\section{Statistical Tests}

I HOPE some space will be afforded me for comment on Prof. Kar] Pearson's letters ${ }^{1}$, since I fear that Prof. Pearson's expressed opinions are not calculated to engender trust in modern statistical methods. These have proved, however, to be of almost universal practical service in minimising the number of times an observer may be misled by his observations.

'To commence with, I must answer Prof. Pearson's attack on the logical position of Prof. Fisher and myself. No doubt Prof. Fisher will have something to say on the matter. For myself, I would point out that I never assume that data are capable of proving either the truth or the untruth of a hypothetical cause of our observations. The hypotheses being tested by the data are the following: Do the data indicate that a stipulated hypothesis is not likely to be true; and the reverse hypothesis-do the data not indicate this? All statistical tests appear to me to be of this kind. If the data do not indicate-I am not using the word prove-that the stipulated. hypothesis is false, the simplest explanation of the data is often that the hypothesis is true. This holds good especially in cases where the stipulated hypothesis is known to be a simple and a likely explanation of the data. Such cases are very numerous.

To attempt to cover all hypothetical frequency distributions under the term 'graduation formulæ' is to overlook the fact that, as used, such distributions are of two radically different kinds. The first kind contains those which may be expected to have given rise to our observations. Of this kind is the binomial distribution as used for testing bias in dice or throwing of dice. The 'limit' approximations, the normal distribution, Prof. Pearson's Type III, the GaltonMacalister, and the Poisson series, for example, may often be justified as 'expected' explanations; also the straight line, the parabola and the hyperbola and the aggregate of two or more samples. The manner in which they arise in theory may be assumed reasonably to resemble the manner in which they arise in practice in many cases. They are reasonable explanations of our data unless these themselves indicate that they are not. They are not mere graduation formulæ. Of the second kind are the distributions mentioned, together with a host of empirical distributions without simple theoretical basis, when they cannot explain the origin or cause of the data. These are truly mere graduation formulæ. They may be very useful as such but do not increase our knowledge of the work of Nature. Of such a kind is Makeham's formula. To use such formulæ to cover a combination of simple explanations of observations is to obscure the workings of Nature, not to elucidate them. Yet as mere graduation formulæ they may be ideal.

Prof. Fisher's school of statisticians aims at disentangling combined simple explanations of observations from each other and thus showing up likely causes of the data. It is true that in their work it is assumed generally that the observations or simple functions of them are samples from a normal population since the processes of analysis used are then more readily applied. It has been shown, however, that these processes of analysis are often fully justified where the fundamental assumption is not strictly justified, and that the methods are most valuable in minimising the number of times an observer may be misled by data. Mere graduation formulæ cannot ever perform this practical service.
My plea for the use of different levels of significance as criteria of judgment whether data do or do not indicate the unlikelihood of a given hypothesis has an analogy in engineering practice. I mean the use of the 'breaking strain' and the 'working Joad' of a given kind of rope. At strains near the breaking strain the rope is likely to break : at strains near the working load it is unlikely to break. At intermediate strains the likelihood of breaking cannot be expressed. With full knowledge the 'likelihood' could be expressed as a probability, but such full knowledge is not assumed. Reasonable safety is what is required. My aim is to make a safety-gap between "not shown to be an unreasonable explanation" and "may be considered a likely explanation". The notion does not apply to graduation formulæ, but only to expected causes. If no particular cause is expected it would appear safer to consider always that the sample exactly represents the form of the population. No other population is shown to be even possible by the sample itself. This does not preclude the use of a graduation formula to express concisely the general form of the sample and to provide parameter-distributions of very similar form to those applying to the sample-population.

H. J. Buchanan-Wollaston.

Fisheries Laboratory, Lowestoft.

Oct. 8.

${ }^{1}$ Nature, 136, 296, 550, Aug. 24 and Oct. 5, 1935.

\section{The Accessibility of Discoveries}

We are indebted to Nature (136, p. 606) for an admirably sharp picture from Dr. Royds setting out his discovery (by its emission spectrum, which is in excess of its absorption as an atmosphere) of a layer of oxygen about a thousand miles deep at the base of the solar chromosphere. As he hints, his graph of intensities gives data for the law of distribution of oxygen-density with height: and he refers to a forthcoming Kodaikanal Bulletin for a discussion of this fundamental problem. But where is one to find it? When I was a member of the Indian Observatories Committee, the Bulletin came to me regularly, and was often of intense observational interest, which I sometimes exploited: but about twenty years ago my place on the Committee was vacated, and I have never seen a Bulletin since, nor do I know how to find one. Yet I am still favoured with personal copies of American and French astronomical reports. I am, moreover, by way of contrast, often overloaded with astronomical theory coming from the Royal Society and the Royal Astronomical Society, in the main too complicated analytically for my slow rate of appreciation, as an amateur no longer young. The brief accounts in Science Abstracts come therefore as a relief.

This note is presented mainly by way of illustration of current methods, especially in Government publications, against which I used to protest in vain when I had a seat in the House of Commons, of fixing prices on a scale that will recover the expense if all the copies of the edition are sold, regardless of the consideration that this price may prohibit the sale of any copies at all. And then what is the use?

Holywood, JoSEPH LARMOR.

Co. Down

Oct. 11 . 